\title{
Characterizing the fecal bacteria and archaea community of heifers and lactating cows through 165 rRNA next-generation sequencing
}

\author{
Filippo Cendron $^{1}$ (D) $\cdot$ Giovanni Niero $^{1} \cdot$ Gabriele Carlino $^{1} \cdot$ Mauro Penasa $^{1} \cdot$ Martino Cassandro $^{1}$
}

Received: 2 April 2020 /Revised: 29 June 2020 / Accepted: 11 August 2020 / Published online: 26 August 2020

(C) The Author(s) 2020

\begin{abstract}
The aim of this study was to describe the fecal bacteria and archaea composition of Holstein-Friesian and Simmental heifers and lactating cows, using 16S rRNA gene sequencing. Bacteria and archaea communities were characterized and compared between heifers and cows of the same breed. Two breeds from different farms were considered, just to speculate about the conservation of the microbiome differences between cows and heifers that undergo different management conditions. The two breeds were from two different herds. Firmicutes, Bacteroidetes, Actinobacteria, and Proteobacteria were the most abundant phyla in all experimental groups. Alpha- and beta-diversity metrics showed significant differences between heifers and cows within the same breed, supported by principal coordinate analysis. The analysis of Holstein-Friesian fecal microbiome composition revealed 3 different bacteria families, 2 genera, and 2 species that differed between heifers and cows; on the other hand, Simmental heifers and cows differed only for one bacteria family, one archaeal genus, and one bacteria species. Results of the present study suggest that fecal communities of heifers and cows are different, and that fecal microbiome is maintained across experimental groups.
\end{abstract}

Keywords Dairy and dual-purpose breeds $\cdot$ Efficiency $\cdot$ Feces $\cdot$ Microbiome

\section{Introduction}

The fecal microbiome consists of a complex community of microorganisms and represents a central issue in relation to cattle welfare and feed efficiency. In particular, the associations between fecal microbiome and animal health have been shown in the intestinal microbiota of calves (Oikonomou et al. 2013). The main factor that influences fecal microbiome composition is animal diet. Callaway et al. (2010) carried out an evaluation of bacterial diversity of 6 cattle (3 Jersey cows and 3 Angus steers) through a comparison of 3 different diets in terms of amount of dried distillers grain; Shanks et al. (2011) analyzed the structure of fecal community in 30 adult beef cattle equally divided in 3 diet groups; and Rice et al. (2012) evaluated the influence of different types and amount of

Communicated by: Maciej Szydlowski

Filippo Cendron

filippo.cendron@unipd.it

1 Department of Agronomy, Food, Natural Resources, Animals and Environment, University of Padova, Viale dell'Università, 16, 35020 Legnaro, PD, Italy distillers grains on fecal microbial assemblages in 20 crossbreed cattle. The forage to concentrates ratio in the diet is the major factor affecting fecal microbiome composition in cattle (Kim et al. 2014). According to the meta-analysis of Kim and Wells (2016), the fecal cattle microbiome is composed of 10 phyla, 17 classes, 28 orders, 59 families, and 100 genera. Firmicutes is the most represented phylum, followed by Bacteroidetes and Proteobacteria. Within Firmicutes, Clostridia and Fecalibacterium are the largest class and genus, respectively. Within Bacteroidetes, Bacteroidia is the largest class and Prevotella the largest genus. Finally, Proteobacteria includes Gammaproteobacteria and Succinivibrio as the most abundant class and genus, respectively. To investigate the microbiome in cattle, most studies have used DNA-based methodologies such as Sanger sequencing technology, quantitative real-time polymerase chain reaction, and phylogenetic microarrays (Kim et al. 2017; Mende et al. 2012). Currently, the next-generation sequencing (NGS) is considered the most reliable approach to evaluate the diversity of bacteria, both in rumen and feces of cattle (Kim et al. 2017). The $16 \mathrm{~S}$ rRNA is widely used as reference gene to determine the composition of bacterial community due to its phylogenetic variability (Tringe and Hugenholtz 2008); indeed, it includes 9 hypervariable regions and 10 conserved 
regions. The conserved regions $(\mathrm{C} 1$ to $\mathrm{C} 10)$ are shared among bacterial and archaeal species, whereas 16S rRNA hypervariable regions (V1 to V9) are different. The latter can be targeted to identify individual bacterial or archaeal species using PCR with species-specific primers for the 16S rRNA gene. Data analysis assigns 16S rRNA sequences to operational taxonomic units (OTUs) that can be identified according to available database. The literature reports differences of fecal microbiome composition within beef cattle breeds, across dairy and beef cattle breeds, and within crossbreed cattle (Durso et al. 2012). Comparisons between heifers and cows of dairy and dual-purpose cattle breeds are currently lacking. The aim of this study was to characterize and analyze the difference of the fecal microbiome community of heifers and cows of dairy and dual-purpose cattle breeds, targeting the hypervariable regions of the bacterial $16 \mathrm{~S}$; moreover, we evaluated if the microbiome composition is conserved between the breeds that underwent to different management and diet composition. Gaining knowledge on these aspects is expected to be beneficial to investigate changes in methane emissions and variation of feed efficiency, as well as to develop non-invasive routine controls to evaluate animal welfare and health.

\section{Materials and methods}

\section{Sample collection and DNA extraction}

Fecal samples were collected through rectal picking during routine health monitoring of animals by authorized veterinarians of the Breeders Association of Veneto Region (Italy). Twenty individual samples (one sample per animal) from 2 single-breed herds (one rearing Holstein-Friesian and the other Simmental breed) were collected for microbiome analysis, considering two categories: cows and heifers. Animals were divided in 2 experimental groups: (1) Holstein-Friesian heifers (HFH, $n=5$ ) and Holstein-Friesian cows (HFC, $n=5$ ); (2) Simmental heifers (SIH, $n=5$ ) and Simmental cows (SIC, $n=$ 5). The sample size was chosen after a literature review (Callaway et al. 2010; Sandri et al. 2018). A description of rations used in the 2 farms is presented in Table 1.

Feces were stored at $-80{ }^{\circ} \mathrm{C}$ within $1 \mathrm{~h}$ from sampling. DNA extraction was performed through AllPrep PowerFecal@ DNA/RNA Kit (Qiagen, Hilden, Germany), and the quantity and quality of total DNA were checked through spectrophotometer assay (Multiscan Sky, Thermo Fisher Scientific, USA).

\section{Next-generation sequencing}

Total genomic DNA was amplified by using a standard protocol and modified primers (Takahashi et al. 2014). Amplicons were purified through magnetic beads Agencourt
Table 1 Diet composition ( $\mathrm{kg} /$ day) in each farm and cattle category

\begin{tabular}{llllll}
\hline & \multicolumn{2}{l}{ Farm 1} & & \multicolumn{2}{l}{ Farm 2 } \\
\cline { 2 - 3 } \cline { 5 - 6 } & HFC & HFH & & SIC & SIH \\
\hline Corn silage & 22 & 8 & & 25 & 7.5 \\
Wheat silage & - & 3.5 & & - \\
Corn mash & 6 & - & & - & - \\
Alfalfa hay & 4.5 & - & & 5 & - \\
Grass hay & 1.5 & 3.5 & & 2 \\
Soybean meal & 3.3 & 1 & & - \\
Soybean flakes & 0.6 & - & & - \\
Corn grain & 2 & - & & - \\
Straw wheat & 0.5 & - & & - \\
Corn meal & - & - & & 5.3 \\
Protein supplement & ${ }^{\mathrm{a}}$ & - & - & & - \\
Mineral-vitamin premix & 0.5 & 0.25 & & 0.5 & - \\
Energetic supplement & $\mathrm{b}$ & - & - & 3.8 & - \\
Hydrogenated fat & 0.3 & - & 0.3 & - \\
Extruded flaxseed & 0.25 & - & - & - \\
Sodium bicarbonate & 0.15 & - & - & - \\
Yeast & - & - & 0.05 & - \\
Total mixed ration & 41.6 & 16.25 & 41.65 & 17.3 \\
\hline
\end{tabular}

$H F C$, Holstein-Friesian cows; HFH, Holstein-Friesian heifers; SIC, Simmental cows; $S I H$, Simmental heifers

${ }^{\text {a }}$ Sunflower seed flour, roasted soybean seeds, dehulled soy flour-based feed, maize, sugar cane molasses

${ }^{\mathrm{b}}$ Maize, barley, sugar cane molasses

XP 0.8× (Beckman Coulter, Brea, CA, USA) and amplified through HiSeq by using Index Nextera XT kit (Illumina, San Diego, CA, USA). All amplified sequences were normalized by SequalPrep (Thermo Fisher, Waltham, MA, USA) and precipitated through magnetic beads Agencourt XP $0.8 \times$. Libraries were loaded onto MiSeq (Illumina, San Diego, CA, USA) and sequenced following V3-300PE strategy.

\section{Statistical analyses}

The OTUs obtained from 16S rRNA sequencing results were filtered for $0.005 \%$ frequency, and organized in an OTU table. The taxonomic survey was obtained from a cross comparison between the QIIME2 software package (http://qiime.org/) and the two databases SILVA v.1.132 and Geengenes v.13.8 (the last as a comparison); clustered OTUs were matched against references from databases. Alpha-diversity analyses were conducted considering Observed OTUs, Shannon Index, Pielou's Evenness, and Faith's Phylogenetic Diversity Index using QIIME2 platform. All sequences were clustered with representative OTUs and cleaned considering $97 \%$ of identity as cutoff. The statistical significance of each index was analyzed 
by Kruskal-Wallis non-parametric test, comparing cows and heifers within the same breed. Beta-diversity was calculated through the Bray-Curtis Metric, Jaccard Metric, and the UniFrac Metric (weighted and unweighted) to evaluate the dissimilarity and distance between the animals of the same breed. Dissimilarities in fecal bacteria and archaea communities were visualized using principal coordinate analysis (PCoA) method. The permutational analysis of variance (PERMANOVA) (Anderson 2005) and analysis of similarities (ANOSIM) were performed in R-vegan package adonis (Oksanen et al. 2017). Finally, differential abundance test was performed using ANCOM packages of R software (Team R, Core Team 2015). Significance was determined through $W$ statistic, which indicates the number of times the null hypothesis was rejected. Positive values of $W$ statistic correspond to more abundant taxa in the comparison of HFH vs HFC, and $\mathrm{SIH}$ vs SIC. The test was performed accounting for the percentile abundance.

\section{Results}

\section{Taxonomic identification}

The total OTUs obtained $(2,302)$ were clustered trough SILVA and Geengenes for the taxonomic analysis; this identified the presence of 2 kingdoms, 14 phyla, 22 classes, 34 orders, 74 families, 212 genera, and 350 species, while the remaining sequences were not assigned to known phyla (Table 2).

Archaea were represented by Euryarchaeota phylum which includes 5 genera equally distributed among the 2 experimental groups (Table 2). Within this phylum, we found several microorganisms that colonize the rumen and are involved in methane production (Holmes and Smith 2016). It is worth noting that the difference regarding Methanosphaera genus was larger for HFC (141 sequences) and SIC (157 sequences) than HFH (22 sequences) and $\mathrm{SIH}$ (74 sequences), likely due to the physiological status and diet composition (Hook et al. 2010). As expected, Bacteria was the largest domain in all experimental groups, representing about twothirds of the total microbiome (Fig. 1).

\section{Abundance of bacterial and archeal communities differs between heifers and lactating cows}

As expected, within bacteria domain, Firmicutes represented the most abundant phylum (Table 2 and Fig. 1). In HFH, Paeniclostridium and Romboutsia were the largest genera, covering 1,543 and 1,119 sequences, respectively, followed by Clostridium, Eubacterium, and Turicibacter. The same order of abundance was maintained in SIH and SIC; however, in HFC, Anaerovibrio (97 sequences), Blautia (137 sequences), Marvinbryantia (157 sequences), Oscillibacter (148 sequences), Roseburia (202 sequences), and Lachnospiraceae AC2044group (274 sequences) showed high abundance. In $\mathrm{SIH}$, the Candidatus class showed good number of sequences (138 sequences), while in SIC Syntrophococcus sequences were greater than the other groups. Moreover, Fourneriella genus was identified only in HFC (13 sequences) and SIC (12 sequences), being probably related to a diet rich in corn. Intestinimonas was represented only in HFH (3 sequences) and SIH (6 sequences).

Bacteroidetes represented the second most abundant phylum with 12,529 sequences in HFH; 10,308 in HFC; 9,997 in $\mathrm{SIH}$; and 10,019 in SIC (Table 2). Prevotellaceae was the largest family and in all groups comprised more than 3,000 sequences, with a particular relative abundance of some genera in heifers, as Prevotellaceae UCG-004, or in cows, as Prevotellaceae UCG-003. Rikenellaceae RC9 gut group was the second largest genus and comprised 2,380 sequences in HFH; 2,015 in HFC; 2,238 in SIH; and 1,918 in SIC. The third largest genus identified was Bacteroides with high differences between heifers and cows; indeed, in HFH (1,715 sequences) and $\mathrm{SIH}$ (1,759 sequences), the abundance was lower compared with HFC (2,807 sequences) and SIC (2,350 sequences). Alistipes was another important genus (Table 2) that showed great variability among the experimental groups: 1,489 sequences in $\mathrm{HFH} ; 866$ in HFC; 1,108 in $\mathrm{SIH}$; and 709 in SIC, and it is typical component of gut microbiome (Xin et al. 2019).

The third largest phylum was Actinobacteria; these Grampositive bacteria have been studied mainly as a minor component of rumen microflora, representing about $3 \%$ of entire community, and this aspect reflect our results (Sulak et al. 2012). This group is involved in the amylase, caseins, gelatinase, lipase, chitins, and cellulose enzyme production (Borsanelli et al. 2018).

\section{Minor phyla}

The incidence of Proteobacteria phylum was variable among the 2 experimental groups. In particular, 251 sequences were detected in HFH, 760 in HFC, 566 in SIH, and 216 in SIC (Table 2). Succinivibrio was the largest genus of Proteobacteria especially in HFC (512 sequences); the high presence of genus Succinivibrio in cows is probably related to an abundant corn-based diet as described by Kim et al. (2014). Mailhella genus was the largest phylotype in heifers (178 sequences in $\mathrm{HFH}$ and 279 in $\mathrm{SIH}$ ); however, their role is almost unknown in cattle and probably their different abundance could be related to animal diet. It is worth noting that the genera of Proteobacteria phylum reported in our study were significantly different from those reported in the study of Kim and Wells (2016). Chloroflexi, Cyanobacteria, Elusimicrobia, Epsilonbacteraeota, Fibrobacteres, and 
Table 2 Average number of sequences (standard deviation) per taxon obtained from fecal samples of Holstein-Friesian heifers (HFH), HolsteinFriesian cows (HFC), Simmental heifers (SIH), and Simmental cows (SIC)

\begin{tabular}{|c|c|c|c|c|}
\hline & $\mathrm{HFH}$ & $\mathrm{HFC}$ & $\mathrm{SIH}$ & $\mathrm{SIC}$ \\
\hline Archaea & $819(371)$ & $2,289(731)$ & $1,429(503)$ & $1,557(539)$ \\
\hline Euryarchaeota & $819(371)$ & $2,289(731)$ & $1,429(503)$ & $1,557(539)$ \\
\hline Methanobrevibacter & $700(277)$ & $2,132(663)$ & $1,200(414)$ & $1,389(532)$ \\
\hline Methanocorpusculum & $73(66)$ & $17(18)$ & $77(38)$ & $11(10)$ \\
\hline Methanosphaera & $22(12)$ & $141(64)$ & $74(33)$ & $157(34)$ \\
\hline Unclassified Methanobacteriaceae & $13(9)$ & - & $73(25)$ & - \\
\hline Uncultured Methanomethylophilaceae & $13(7)$ & - & $6(5)$ & - \\
\hline Bacteria & 27,662 & 26,443 & 26,401 & 27,262 \\
\hline Actinobacteria & $35(16)$ & $1,265(589)$ & $73(41)$ & $594(399)$ \\
\hline Aeriscardovia & - & $14(3)$ & - & $3(4)$ \\
\hline Arcanobacterium & $6(7)$ & - & $1(2)$ & - \\
\hline Atopobium & $3(4)$ & $5(1)$ & $3(4)$ & $9(8)$ \\
\hline Bifidobacterium & - & $1,130(565)$ & - & $28(26)$ \\
\hline Olsenella & - & $66(42)$ & $25(34)$ & $493(357)$ \\
\hline Raoultibacter & $2(3)$ & - & $7(5)$ & - \\
\hline Eggerthellaceae DNF00809 & $10(11)$ & $13(6)$ & $22(14)$ & $13(10)$ \\
\hline Uncultured Eggerthellaceae & $1(2)$ & $5(6)$ & $2(3)$ & $13(8)$ \\
\hline Unclassified Actinobacteria & $12(7)$ & $31(16)$ & $14(20)$ & $35(20)$ \\
\hline Bacteroidetes & $12,529(610)$ & $10,308(753)$ & $9,997(586)$ & $10,019(581)$ \\
\hline Alistipes & $1,489(181)$ & $866(207)$ & $1,108(248)$ & $709(230)$ \\
\hline Alloprevotella & $202(72)$ & $198(146)$ & $135(86)$ & $780(348)$ \\
\hline Bacteroides & $1,715(67)$ & $2,807(151)$ & $1,759(263)$ & $2,350(230)$ \\
\hline Odoribacter & $32(8)$ & $16(24)$ & $20(11)$ & $35(16)$ \\
\hline Sanguibacteroides & $2(4)$ & - & $7(6)$ & - \\
\hline Paludibacter & - & $15(22)$ & - & - \\
\hline Parabacteroides & $11(6)$ & $37(7)$ & $19(10)$ & $103(23)$ \\
\hline Prevotella & $137(39)$ & $479(113)$ & $78(29)$ & $193(123)$ \\
\hline Prevotellaceae UCG-001 & $82(32)$ & $258(50)$ & $74(30)$ & $150(58)$ \\
\hline Prevotellaceae UCG-003 & $988(76)$ & $1,908(100)$ & $704(160)$ & $1,172(243)$ \\
\hline Prevotellaceae UCG-004 & $1,168(151)$ & $183(147)$ & $1,308(264)$ & $532(81)$ \\
\hline Prevotellaceae Ga6A1 group & $6(7)$ & $25(21)$ & $18(14)$ & $33(27)$ \\
\hline Rikenellaceae RC9 gut group & $2,380(158)$ & $2,015(254)$ & $2,238(313)$ & $1,918(229)$ \\
\hline Rikenellace dgA-11 gut group & $952(183)$ & $70(83)$ & $622(88)$ & $132(79)$ \\
\hline Uncultured Bacteroidales F082 & $558(120)$ & $8(12)$ & $773(117)$ & $87(38)$ \\
\hline Uncultured Bacteroidales gir-aah93h0 & $335(116)$ & $235(233)$ & $177(32)$ & $335(240)$ \\
\hline Uncultured Bacteroidales M2PB4-65 termite group & $169(183)$ & - & $234(192)$ & - \\
\hline Uncultured Bacteroidales p-251-o5 & $118(65)$ & $21(41)$ & $169(81)$ & $125(123)$ \\
\hline Uncultured Bacteroidales p-2534-18B5 gut group & $398(185)$ & - & - & $235(216)$ \\
\hline Uncultured Bacteroidales RF16 group Paludibacter sp. & $36(20)$ & - & $30(28)$ & $19(6)$ \\
\hline Uncultured Bacteroidales RF16 group Porphyromonadaceae bacterium & $225(38)$ & $196(102)$ & $259(179)$ & $218(167)$ \\
\hline Uncultured Barnesiellaceae & $29(11)$ & $46(23)$ & $21(7)$ & $61(44)$ \\
\hline Uncultured Bacteroidales RF16 group Uncultured bacterium & $43(16)$ & $22(22)$ & $165(163)$ & $35(23)$ \\
\hline Uncultured Bacteroidales UCG-001 & $49(10)$ & - & $76(17)$ & $25(9)$ \\
\hline Uncultured Bacteroidales & - & - & $31(8)$ & $11(14)$ \\
\hline Uncultured Dysgonomonadaceae & $149(65)$ & $82(95)$ & $316(208)$ & $198(129)$ \\
\hline Uncultured Flavobacteriaceae & $702(130)$ & $394(77)$ & $163(53)$ & $332(106)$ \\
\hline Uncultured Muribaculaceae & $213(139)$ & $60(34)$ & $31(9)$ & $17(11)$ \\
\hline Unclassified Bacteroidetes & $341(91)$ & $367(326)$ & $166(93)$ & $195(106)$ \\
\hline
\end{tabular}


Table 2 (continued)

\begin{tabular}{|c|c|c|c|c|}
\hline & $\mathrm{HFH}$ & $\mathrm{HFC}$ & $\mathrm{SIH}$ & $\mathrm{SIC}$ \\
\hline Chloroflexi & $3(4)$ & & $34(12)$ & \\
\hline Flexilinea & $3(4)$ & - & $34(12)$ & - \\
\hline Cyanobacteria & $396(174)$ & $147(212)$ & 439 (146) & $140(84)$ \\
\hline Uncultured Cyanobacteria & $371(162)$ & $147(212)$ & $423(162)$ & $140(84)$ \\
\hline Unclassified Cyanobacteria & $24(25)$ & - & $16(18)$ & - \\
\hline Elusimicrobia & $32(20)$ & $3(6)$ & $39(31)$ & $2(4)$ \\
\hline Eluisimicrobium & $32(20)$ & $3(6)$ & $39(31)$ & $2(4)$ \\
\hline$\underline{\text { Epsilonbacteraeota }}$ & $7(16)$ & $1(1)$ & $2(4)$ & $1(3)$ \\
\hline Campylobacter & $7(16)$ & $1(1)$ & $2(4)$ & $1(3)$ \\
\hline Fibrobacteres & $156(51)$ & - & $511(149)$ & $71(70)$ \\
\hline Fibrobacter & $156(51)$ & - & $511(149)$ & $71(70)$ \\
\hline Firmicutes & $13,285(1130)$ & $13,568(546)$ & $13,216(869)$ & $15,380(1022)$ \\
\hline Acetitomaculum & $17(14)$ & $47(21)$ & $65(12)$ & $65(10)$ \\
\hline Aerococcus & $6(7)$ & $3(8)$ & - & - \\
\hline Agathobacter & $10(14)$ & $170(78)$ & - & $77(70)$ \\
\hline Anaerorhabdus furcosa group & $28(14)$ & $25(13)$ & $17(10)$ & $20(7)$ \\
\hline Anaerosporobacter & $51(21)$ & $18(17)$ & $71(98)$ & $20(31)$ \\
\hline Anaerostipes & - & $22(27)$ & - & - \\
\hline Anaerovibrio & - & 97 (107) & - & $29(30)$ \\
\hline Anaerovorax & $30(11)$ & $16(14)$ & $42(8)$ & $200(52)$ \\
\hline Angelakisella & $19(4)$ & - & $8(3)$ & - \\
\hline Blautia & $7(10)$ & $137(59)$ & $24(14)$ & $104(37)$ \\
\hline Breznakia & $1(2)$ & $21(16)$ & $1(1)$ & $3(3)$ \\
\hline Butyricicoccus & $5(12)$ & $7(4)$ & - & $6(4)$ \\
\hline Butyrivibrio & $8(8)$ & $19(12)$ & $2(3)$ & $12(12)$ \\
\hline Candidatus Soleaferrea & $107(20)$ & $42(14)$ & $138(29)$ & $90(10)$ \\
\hline Candidatus Stoquefichus & - & $149(121)$ & - & - \\
\hline Caproiciproducens & $7(9)$ & $1(1)$ & $11(10)$ & $8(8)$ \\
\hline Cellulosilyticum & $52(69)$ & $31(23)$ & $5(4)$ & $64(33)$ \\
\hline Christensenellaceae $R-7$ group & 498 (134) & 765 (213) & 797 (156) & $905(403)$ \\
\hline Clostridium & $173(11)$ & $307(271)$ & $41(14)$ & $482(262)$ \\
\hline Coprobacillus & $1(3)$ & $42(9)$ & $8(3)$ & $6(8)$ \\
\hline Coprococcus & $17(14)$ & $121(53)$ & $37(40)$ & $122(61)$ \\
\hline Defluvitaleaceae UCG-011 & $22(9)$ & $14(8)$ & $8(9)$ & $13(11)$ \\
\hline Dielma & $7(5)$ & $13(8)$ & $12(7)$ & $15(3)$ \\
\hline Dorea & $56(30)$ & $90(34)$ & $71(23)$ & $78(39)$ \\
\hline Eisenbergiella & - & $4(5)$ & $1(1)$ & $2(5)$ \\
\hline Erysipelatoclostridium & $1(3)$ & $42(9)$ & $8(3)$ & $42(12)$ \\
\hline Erysipelotrichaceae UCG-002 & - & $12(16)$ & - & - \\
\hline Erysipelotrichaceae UCG-004 & $10(14)$ & $104(41)$ & - & $24(30)$ \\
\hline Erysipelotrichaceae UCG-006 & - & - & - & $16(17)$ \\
\hline Erysipelotrichaceae UCG-007 & - & - & - & $27(18)$ \\
\hline Erysipelotrichaceae UCG-009 & - & $4(3)$ & - & - \\
\hline Eubacterium brachy group & $56(31)$ & $66(10)$ & $72(8)$ & $80(21)$ \\
\hline Eubacterium halii group & $7(7)$ & $1(1)$ & $22(5)$ & $37(33)$ \\
\hline Eubacterium nodatum group & $42(35)$ & $31(10)$ & $80(22)$ & $71(43)$ \\
\hline Eubacteriu ruminantium group & $2(4)$ & $23(22)$ & $11(11)$ & $32(22)$ \\
\hline Eubacterium ventriosum group & $2(2)$ & - & $11(8)$ & - \\
\hline Fecalibacterium & - & $5(2)$ & - & $4(2)$ \\
\hline
\end{tabular}


Table 2 (continued)

\begin{tabular}{|c|c|c|c|c|}
\hline & $\mathrm{HFH}$ & HFC & $\mathrm{SIH}$ & SIC \\
\hline Fecalitalea & $9(8)$ & $65(51)$ & $2(3)$ & $7(7)$ \\
\hline Family XIII AD3011 & $273(50)$ & $140(18)$ & $273(26)$ & $200(52)$ \\
\hline Family XIII UCG-001 & $3(4)$ & $6(2)$ & $3(4)$ & $10(2)$ \\
\hline Flavonifractor & $14(32)$ & $66(36)$ & $30(19)$ & $57(21)$ \\
\hline Fourneriella & - & $13(10)$ & - & $12(3)$ \\
\hline Howardella & $4(6)$ & $20(8)$ & $18(10)$ & $45(33)$ \\
\hline Intestinimonas & $3(7)$ & - & $6(6)$ & - \\
\hline Lachnoclostridium & $51(35)$ & $24(29)$ & $55(16)$ & $44(24)$ \\
\hline Lachnospira & $8(19)$ & - & - & - \\
\hline Lachnospiraceae AC2044 group & $48(20)$ & $274(118)$ & $44(18)$ & $68(70)$ \\
\hline Lachnospiraceae FCS020 group & $5(5)$ & $5(7)$ & $7(8)$ & - \\
\hline Lachnospiraceae FE2018 group & - & $4(3)$ & $2(3)$ & $2(2)$ \\
\hline Lachnospiraceae NK3A20 group & $67(32)$ & $149(52)$ & $87(29)$ & $154(53)$ \\
\hline Lachnospiracea NK4A136 group & $9(13)$ & $175(133)$ & $184(105)$ & $228(133)$ \\
\hline Lachnospiraceae UCG-001 & $36(16)$ & $62(51)$ & $35(12)$ & $46(11)$ \\
\hline Lachnospiraceae UCG-002 & - & - & - & $5(4)$ \\
\hline Lachnospiraceae UCG-007 & - & - & - & $9(13)$ \\
\hline Lachnospiraceae UCG-010 & $106(29)$ & $58(18)$ & $101(29)$ & $154(68)$ \\
\hline Lysinibacillus & $2(4)$ & - & $3(7)$ & $11(1)$ \\
\hline Marvinbryantia & $16(10)$ & $157(54)$ & $27(11)$ & $158(44)$ \\
\hline Mitsuokella & - & $10(10)$ & - & - \\
\hline Mogibacterium & $9(6)$ & $3(7)$ & $12(8)$ & $4(8)$ \\
\hline Moryella & $4(4)$ & $7(4)$ & $7(5)$ & $10(5)$ \\
\hline Negativibacillus & $24(13)$ & $51(12)$ & $30(10)$ & $66(15)$ \\
\hline Oscillibacter & $112(26)$ & $148(25)$ & $86(15)$ & $110(22)$ \\
\hline Oscillospira & $66(28)$ & $1(2)$ & $14(5)$ & $1(2)$ \\
\hline Paeniclostridium & $1,543(261)$ & $433(191)$ & 705 (472) & $370(174)$ \\
\hline Papillibacter & $11(11)$ & $4(0)$ & $14(5)$ & $3(0)$ \\
\hline Phascolarctobacterium & $114(41)$ & $155(31)$ & $107(18)$ & $136(42)$ \\
\hline Pseudobutyrivibrio & $9(20)$ & - & - & - \\
\hline Pygmaiobacter & $29(17)$ & $69(39)$ & $10(6)$ & $56(28)$ \\
\hline Romboutsia & $1,119(206)$ & $514(370)$ & $902(462)$ & $952(423)$ \\
\hline Roseburia & $17(10)$ & $202(257)$ & $10(8)$ & $71(91)$ \\
\hline Ruminiclostridium & $72(29)$ & $15(10)$ & $75(28)$ & $7(2)$ \\
\hline Ruminococcaceae Eubacterium coprostanoligenes group & $795(141)$ & $535(250)$ & $880(109)$ & $928(214)$ \\
\hline Ruminococcace GCA-90066225 & $8(4)$ & $17(10)$ & $10(6)$ & $13(6)$ \\
\hline Ruminococcace NK4A214 group & $217(49)$ & $123(38)$ & $275(33)$ & $187(33)$ \\
\hline Ruminococcaceae UCG-002 & $91(25)$ & $18(13)$ & $104(29)$ & $34(20)$ \\
\hline Ruminococcaceae UCG-004 & $58(15)$ & $45(12)$ & $59(15)$ & $60(20)$ \\
\hline Ruminococcaceae UCG-005 & $2,144(288)$ & $2,913(396)$ & $2,214(395)$ & $3,114(387)$ \\
\hline Ruminococcaceae UCG-009 & $209(39)$ & $84(38)$ & $238(55)$ & $122(22)$ \\
\hline Ruminococcaceae UCG-010 & $1,881(181)$ & $978(439)$ & $1,736(192)$ & $1,044(525)$ \\
\hline Ruminococcaceae UCG-011 & $26(17)$ & - & $110(12)$ & - \\
\hline Ruminococcaceae UCG-013 & $1,156(150)$ & $543(274)$ & $822(83)$ & $941(218)$ \\
\hline Ruminococcaceae UCG-014 & $302(83)$ & $343(347)$ & $360(44)$ & $363(76)$ \\
\hline Ruminococcu gauvreauii group & $2(5)$ & $30(24)$ & $7(9)$ & $77(27)$ \\
\hline Saccharofermentans & $10(10)$ & $3(2)$ & $36(7)$ & $11(6)$ \\
\hline Sharpea & - & $16(17)$ & - & $18(9)$ \\
\hline Solobacterium & - & - & $11(8)$ & - \\
\hline
\end{tabular}


Table 2 (continued)

\begin{tabular}{|c|c|c|c|c|}
\hline & $\mathrm{HFH}$ & $\mathrm{HFC}$ & $\mathrm{SIH}$ & SIC \\
\hline Streptococcus & $1(3)$ & $2(2)$ & $4(4)$ & $1(1)$ \\
\hline Syntrophococcus & $2(4)$ & $4(10)$ & $11(12)$ & $288(229)$ \\
\hline Terrisporobacter & $39(17)$ & $18(0)$ & $18(7)$ & - \\
\hline Turicibacter & $141(19)$ & $254(273)$ & $166(70)$ & $117(100)$ \\
\hline Tyzzerella 4 & $29(17)$ & $107(87)$ & $67(21)$ & $116(32)$ \\
\hline XBB1006 & - & - & $7(8)$ & - \\
\hline Uncultured Christensenellaceae & - & - & $8(6)$ & - \\
\hline Uncultured Clostridiales vadinBB60 group & $99(26)$ & $188(202)$ & $69(37)$ & $179(108)$ \\
\hline Uncultured Erysipelotrichaceae & $12(9)$ & $25(33)$ & - & $16(11)$ \\
\hline Uncultured Lachnospiraceae & $42(18)$ & $92(47)$ & $62(12)$ & $42(6)$ \\
\hline Uncultured Peptococcaceae & $150(20)$ & $64(13)$ & $150(55)$ & $48(16)$ \\
\hline Uncultured Ruminococcaceae & $239(35)$ & $92(23)$ & $252(48)$ & $115(40)$ \\
\hline Uncultured Veillonellaceae & - & - & - & $14(7)$ \\
\hline Unclassified Firmicutes & $1,019(296)$ & $2,092(722)$ & $1,128(302)$ & $1,882(953)$ \\
\hline Patescibacteria & $62(7)$ & $37(13)$ & $83(23)$ & $113(40)$ \\
\hline Candidatus Saccharimonas & $62(7)$ & $37(13)$ & $83(23)$ & $113(40)$ \\
\hline$\underline{\text { Proteobacteria }}$ & $351(108)$ & $760(456)$ & $566(197)$ & $216(144)$ \\
\hline Escherichia-Shigella & - & $39(24)$ & - & $6(9)$ \\
\hline Kingella & $3(5)$ & $6(13)$ & - & - \\
\hline Mailhella & $178(60)$ & $19(9)$ & $279(47)$ & $20(13)$ \\
\hline Parasutterella & $47(16)$ & $52(24)$ & $23(9)$ & $28(11)$ \\
\hline Ruminobacter & $3(4)$ & $54(27)$ & $25(37)$ & $16(28)$ \\
\hline Succinivibrio & $9(21)$ & $512(446)$ & $1(1)$ & $117(93)$ \\
\hline Succinivibrionaceae UCG-001 & - & $5(4)$ & - & - \\
\hline Sutterella & - & $9(4)$ & - & $3(8)$ \\
\hline Uncultured Paracaedibacteraceae & $6(2)$ & - & - & - \\
\hline Uncultured Rhodospirillales & $63(33)$ & - & $168(133)$ & $16(24)$ \\
\hline Uncultured Rickettsiales & $6(4)$ & $7(2)$ & - & - \\
\hline Unclassified Proteobacteria & $36(24)$ & - & $70(80)$ & $10(14)$ \\
\hline$\underline{\text { Spirochaetes }}$ & $197(44)$ & $133(89)$ & $143(35)$ & $263(129)$ \\
\hline Sediminisprirochaeta & $10(8)$ & - & - & - \\
\hline Spirochaetaceae GWE-31-10 & $20(13)$ & $9(15)$ & $14(6)$ & $37(9)$ \\
\hline Treponema & $167(32)$ & $124(75)$ & $129(29)$ & $226(126)$ \\
\hline$\underline{\text { Tenericutes }}$ & $128(45)$ & 139 (19) & $88(37)$ & $84(26)$ \\
\hline Anaeroplasma & - & $40(15)$ & - & $9(10)$ \\
\hline Uncultured Tenericutes & $113(53)$ & $82(70)$ & $70(67)$ & $58(39)$ \\
\hline Unclassified Tenericutes & $15(10)$ & $17(20)$ & $18(24)$ & $17(17)$ \\
\hline$\underline{\text { Verrucomicrobia }}$ & $481(96)$ & $82(113)$ & $850(195)$ & $379(122)$ \\
\hline Akkermansia & $481(96)$ & $82(113)$ & $850(195)$ & $379(122)$ \\
\hline
\end{tabular}

Verrucomicrobia comprised the classes that showed differences in number of sequences among the experimental groups (Table 2). Indeed, their abundance was higher in heifers than cows. Chloroflexi phylum was observed only in HFH (3 sequences) and SIH (34 sequences) due to contamination, since this phylum includes environmental photosynthetic bacteria (Borsanelli et al. 2018). Elusimicrobia and Epsilonbacteraeota are 2 phyla not commonly present in cattle feces (Kim et al.
2017), while Cyanobacteria have been reported in other species, but their role in cattle remains still unknown (Shepherd et al. 2012). Fibrobacteres phylum belongs to the group of bacteria that colonize the rumen; their function in cattle is related to fiber digestion, and thus, their presence is associated to a diet rich in forage (AlZahal et al. 2017). Verrucomicrobia phylum was the most variable among the experimental groups. Indeed, we found 481 sequences in HFH, 82 in HFC, 850 in SIH, and 379 in SIC 

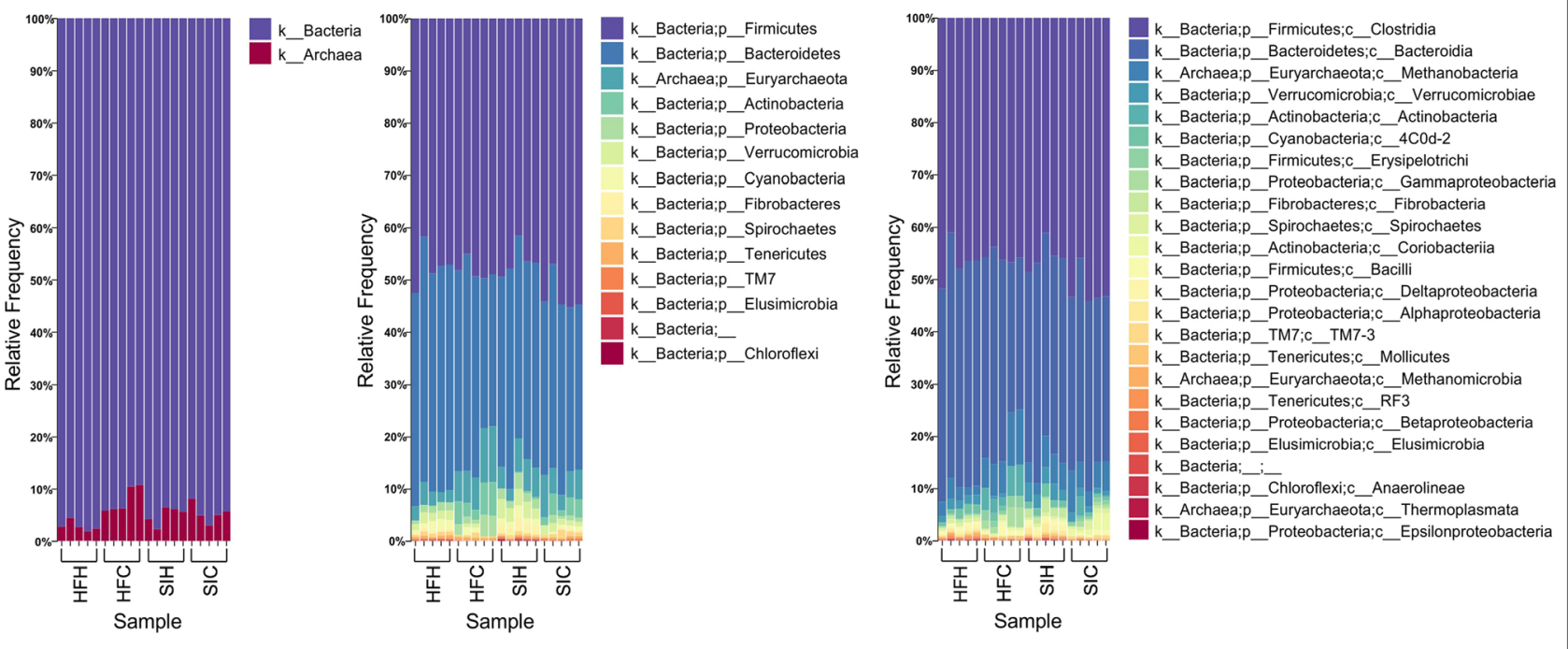

Fig. 1 Next-generation sequencing (NGS) relative abundance at the kingdom, phylum, and class levels. The figure represents 5 experimental replicates for each experimental group ( $x$-axis). Annotation was done using SILVA database: kingdoms are shown on the left side of the figure, phyla on the central part, and classes on the right side
(Table 2). These bacteria are usually not affected by the different diets or sample fractions and their role is still unknown (Deusch et al. 2017). Patescibacteria and Spirochaetes are commonly present in feces of cattle (Nyonyo et al. 2014); indeed, their level has been considered constant among the experimental groups (Table 2). Patescibacteria function is still unknown, while Spirochaetes phylum is associated with the cellulolytic activity (Nyonyo et al. 2014).

\section{Alpha-diversity}

Results of $\alpha$-diversity test are shown in Table 3 . The number of OTUs for all experimental groups ranged from 676 to 798; in particular, the average number of identified OTUs \pm SD in HFH, HFC, SIH, and SIC was $798 \pm 144,676 \pm 75,733 \pm 88$, and $716 \pm 75$, respectively. The number of observed OTUs was not statistically different among samples of the same breed, whereas significant differences were observed for the other indexes. Pielou's Evenness Index showed differences ( $p$ $<0.05)$ between cows and heifers of the same breed: HFH $(0.915 \pm 0.003)$ vs HFC $(0.883 \pm 0.016)$, and SIH $(0.918 \pm$ $0.008)$ vs SIC $(0.899 \pm 0.007)$, related to a different abundance of species, due to the diet composition and animal category. Through Shannon Index the observed number of species was different only between HFH $(32.970 \pm 2.997)$ and HFC $(28.557 \pm 2.228)(p<0.05)$. Faith's Phylogenetic Diversity Index underlined that just the comparison between $\mathrm{HFH}$ $(8.799 \pm 0.241)$ and HFC $(8.299 \pm 0.234)$ was statistically significant $(p<0.05)$, proving that within Holstein-Friesian breed, the microbial species seem to have different phylogenetic taxon. Finally, the OTU table was normalized according to the OTU abundance across samples, and the results were clustered as the heatmap to provide a better pattern across the experimental groups (Fig. 2).

\section{Beta-diversity}

Results of $\beta$-diversity were computed on the rarefied OTU table using different metrics (Table 4). The Bray-Curtis Metric showed high dissimilarity across experimental groups of the same breed, where the index was weakly high in the comparison HFH vs HFC (0.74), and SIH vs SIC (0.64). The PERMANOVA test underlined that the difference was statistically different between the samples of the same breed (HFH vs HFC and SIH vs SIC; $p<0.05)$. The Jaccard Metric provides the difference in the microbial composition among the experimental groups, HFH vs HFC (0.80) and SIH vs SIC (0.78), underlying a significant difference in fecal microbial composition $(p<0.05)$. The UniFrac Metric was considered unweighted and weighted (Table 4). The UniFrac Metric was low in the comparisons HFH vs HFC (unweighted 0.51 and weighted 0.23 ) and SIH vs SIC (unweighted 0.48 and weighted 0.21). Such results were supported by the statistical significance of the PERMANOVA test $(p<0.05)$ in inter-breed comparisons.

PCoA of the Bray-Curtis, Jaccard, and unweighted UniFrac Metrics revealed that microbiota in fecal samples were clustered in 4 different groups identified as our 2 
Table 3 Summary of 16S rRNA OTU data and $\alpha$-diversity values (Evenness Index, Shannon Index, and Faith's Phylogenetic Diversity Index) in association with statistical test

\begin{tabular}{|c|c|c|c|c|c|c|c|c|c|c|c|c|}
\hline & \multicolumn{3}{|c|}{ Observed OTUs } & \multicolumn{3}{|c|}{ Evenness Index } & \multicolumn{3}{|c|}{ Shannon Index } & \multicolumn{3}{|c|}{ Faith PD Index } \\
\hline & Number & SD & & Pielou_e & SD & & Value & $\mathrm{SD}$ & & Value & SD & \\
\hline $\mathrm{HFC}$ & 676 & 75 & & 0.883 & 0.016 & & 28.557 & 2.228 & & 8.299 & 0.234 & \\
\hline $\mathrm{HFH}$ & 798 & 144 & & 0.915 & 0.003 & & 32.970 & 2.997 & & 8.799 & 0.241 & \\
\hline SIC & 716 & 85 & & 0.899 & 0.007 & & 31.547 & 1.685 & & 8.521 & 0.190 & \\
\hline $\mathrm{SIH}$ & 733 & 88 & & 0.918 & 0.008 & & 31.916 & 1.421 & & 8.725 & 0.180 & \\
\hline \multicolumn{13}{|c|}{ Kruskal-Wallis (pairwise) } \\
\hline & $\mathrm{H}$ & $p$ value & $q$ value & $\mathrm{H}$ & $p$ value & $q$ value & $\mathrm{H}$ & $p$ value & $q$ value & $\mathrm{H}$ & $p$ value & $q$ value \\
\hline HFC vs HFH & 2.45 & 0.11 & 0.69 & 6.81 & 0.009 & 0.013 & 4.18 & 0.028 & 0.084 & 4.81 & 0.028 & 0.084 \\
\hline SIC vs SIH & 0.99 & 0.75 & 0.75 & 6.81 & 0.009 & 0.013 & 2.45 & 0.117 & 0.140 & 0.098 & 0.754 & 0.754 \\
\hline
\end{tabular}

Each index is reported as mean \pm standard deviation of the calculated value for each animal within the experimental group. The statistical comparison was considered only between the experimental groups of the same breed through Kruskal-Wallis test

$H F C$, Holstein-Friesian cows; HFH, Holstein-Friesian heifers; SIC, Simmental cows; SIH, Simmental heifers

experimental groups (Bray-Curtis-PC1: $38.12 \%, \mathrm{PC} 2$ : 12.97\%, PC3: 9.78\%; Jaccard-PC1: $30.65 \%$, PC2: 12.72\%, PC3: $10.44 \%$; unweighted UniFrac-PC1: $42.75 \%$, PC2: $12.23 \%$, PC3: $8.31 \%$ ); the repeatability of the results of the 5 animals within the same group was very high, particularly among the HFH and SIH groups (Fig. 3). PCoA of the weighted UniFrac distance revealed that microbiota of the animals of the same group had high variability (weighted UniFrac-PC1: $58.53 \%$, PC2: 11.55\%, PC3: 8.36\%) (Fig. 3).

\section{Abundance of species in comparative context: Holstein-Friesian heifers vs Holstein-Friesian cows and Simmental heifers vs Simmental cows}

The analysis of composition of microbiomes (ANCOM) (Fig. 4) and volcano plot were performed in order to identify the relative abundance of species that was significantly different in the comparisons HFH vs HFC and SIH vs SIC (Kuczynski et al. 2012).
Fig. 2 Hierarchical clustering of core OTUs in the 4 experimental groups, divided by quadrants. All core OTUs were clustered among the animals of the experimental groups. The blue background represents 0 counts, whereas red color indicates higher counts for that particular taxonomic unit in a specific sample

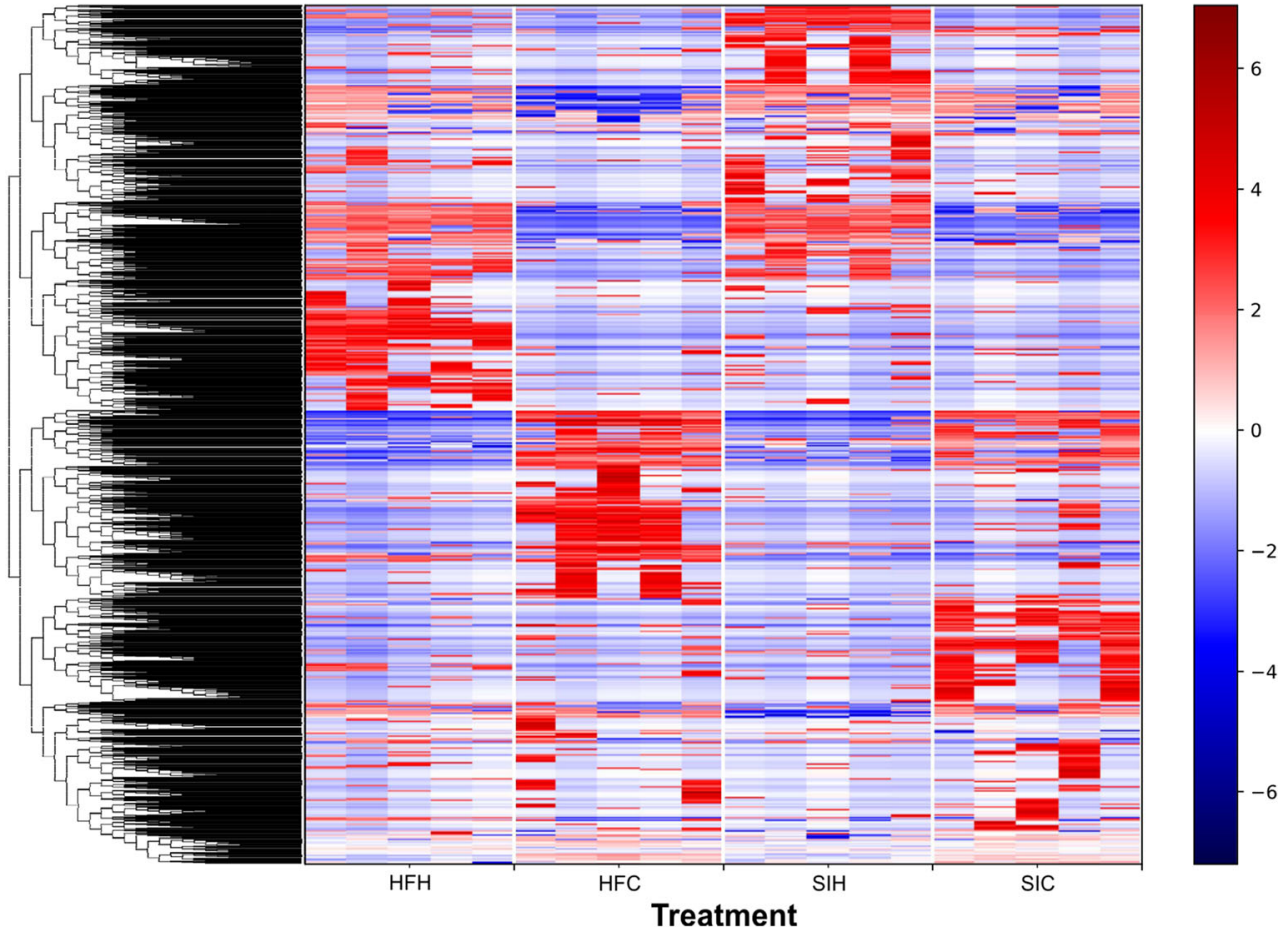


Table $4 \beta$-Diversity values through Bray-Curtis, Jaccard, and unweighted and weighted UniFrac Metrics. $P$ value for each comparison was obtained from PERMANOVA and considered significant at $p<0.05$

\begin{tabular}{lcllll}
\hline & Value & Pairwise PERMANOVA result & Pseudo $F$ & $p$ value & $q$ value \\
\hline Bray-Curtis & & & & & \\
HFH-HFC & 0.74 & HFC vs HFH & 10.9 & 0.009 & 0.013 \\
SIH-SIC & 0.64 & SIC vs SIH & 7.2 & 0.009 & 0.013 \\
Jaccard & & & & & \\
HFH-HFC & 0.80 & HFC vs HFH & 7.46 & 0.011 & 0.013 \\
SIH-SIC & 0.78 & SIC vs SIH & 5.75 & 0.008 & 0.012 \\
Unweighted UniFrac & & & & \\
HFH-HFC & 0.51 & HFC vs HFH & 10.93 & 0.009 & 0.016 \\
SIH-SIC & 0.48 & SIC vs SIH & 8.28 & 0.012 & 0.016 \\
Weighted UniFrac & & & & \\
HFH-HFC & 0.23 & HFC vs HFH & 17.27 & 0.012 & 0.014 \\
SIH-SIC & 0.21 & SIC vs SIH & 7.21 & 0.004 & 0.014 \\
\hline
\end{tabular}

$H F C$, Holstein-Friesian cows; $H F H$, Holstein-Friesian heifers; SIC, Simmental cows; SIH, Simmental heifers. Pvalue for each comparison was considered significant at $p<0.05$.
The comparison between HFH and HFC associated with the ANCOM statistical analysis detected significant differences for 3 bacterial families according to Holm-Bonferroni post hoc test $(p<0.05)$; Anaeroplasmataceae (W 54) and Bifidobacteriaceae (W 52) were the most important families in HFC, and p-2534-18B5 (W 50) in HFH (Fig. 4). The a

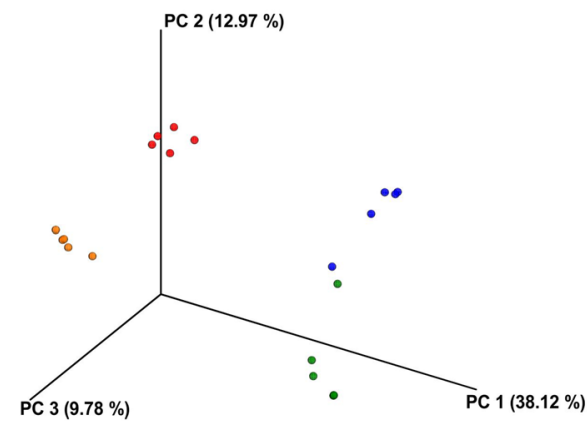

Bray Curtis PCoA

C

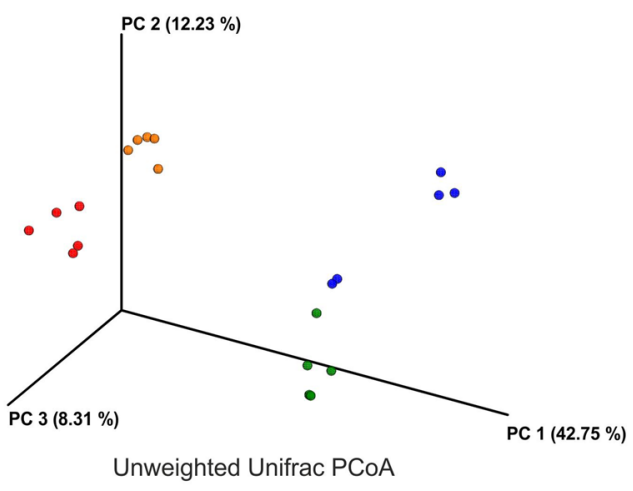

Fig. 3 Principal coordinate analysis (PCoA) of $16 \mathrm{~S}$ metagenomics data of microbial population in feces of Holstein-Friesian and Simmental heifers and cows. Comparison between community diversity based on different metric distances of microbial communities in cattle feces: a

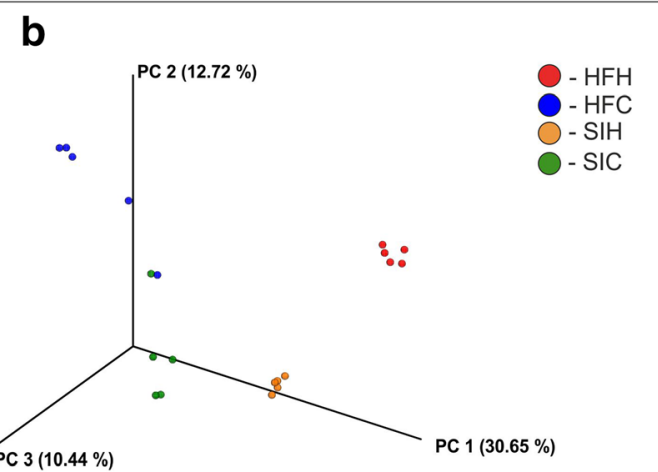

Jaccard PCoA

d

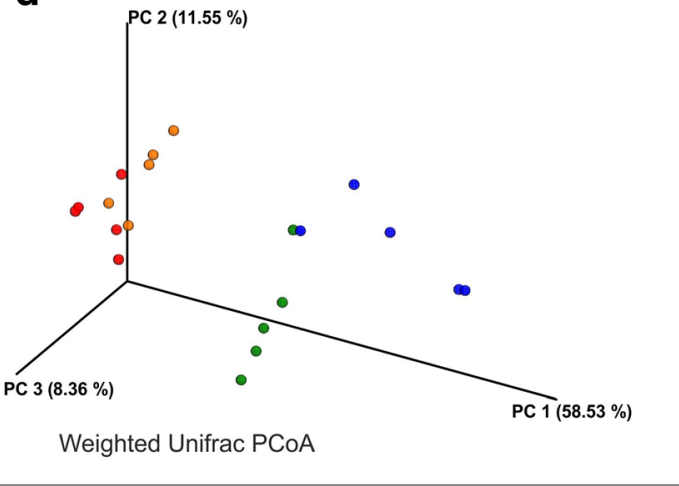

Bray-Curtis; b Jaccard; c unweighted UniFrac; d weighted UniFrac. Experimental groups are HFH (red), HFC (blue), SIH (orange), and SIC (green), each including 5 animals 

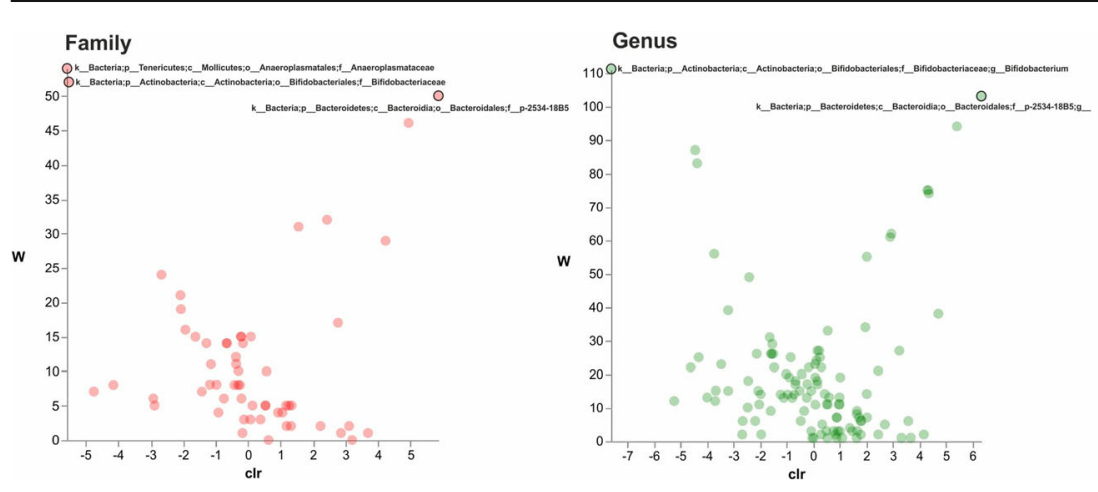

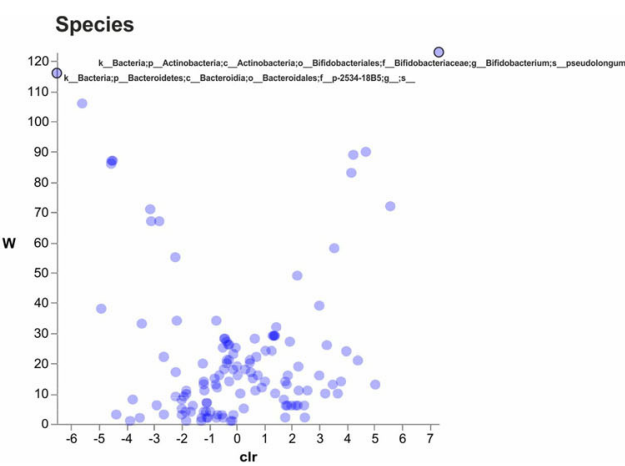

Fig. 4 Volcano plot representation of ANCOM analyses. The horizontal axis is the centered log ratio transformation (clr) representative of the difference in abundance of a given taxonomical unit between HolsteinFriesian cows and Holstein-Friesian heifers at the family (red dots), genus (green dots), and species (blue dots) level. The W statistic indicates the value of the statistical test corresponding to the number of times the null hypothesis was rejected for each taxonomical class. Taxonomical classes with relative abundance significantly different (Holm-Bonferroni corrected $p$ value $<0.05$ ) are evidenced at the top of volcano plot and their taxonomy is reported. A darker shade of color indicates an overlap of OTUs statistical analysis of genera abundance in HFH vs HFC showed significant differences $(p<0.05)$ for Bifidobacterium (Bifidobacteriaceae) (W 103) and for one unclassified genus (p-2534-18B5). Results of the lowest taxonomic level revealed significant differences of species $(p<$ 0.05 ) between HFH and HFC, suggesting a greater presence of Bifidobacterium pseudolongum (W 123) in HFC and p25434-18B unclassified bacterium (W 116) in HFH.

In Simmental breed, Anaerolineales family (W 43) and 1 unclassified Methanobacteriaceae genus (W 96) and species (W 101) showed significant differences $(p<0.05)$ when comparing heifers and cows (Fig. 5). Anaerolinaceae family was more abundant in histidine-fed heifers (Klevenhusen et al. 2017). Indeed, SIH showed more abundant archaeal Methanocorpusculaceae and bacterial Elusimicrobiaceae family. As reported for Holstein-Friesian breed, Methanocorpuscolaceae role in rumen has not been studied yet and the presence in feces could be related to a shift outside from the rumen.

\section{Discussion}

In the present study, we assessed the diversity of microbiome in cow and heifer feces within Holstein-Friesian and Simmental cattle breeds. The 16S rRNA sequencing approach was used to perform analysis of bacterial communities. The members of Firmicutes, Bacteroidetes, Proteobacteria, and Actinobacteria phyla were the most abundant and showed great variability among experimental groups (Kim et al. 2014). The presence of genus Fecalibacterium in the HFC and SIC might be related to a high corn percentage in the diet fed to cows compared with the diet fed to heifers. This hypothesis is corroborated by the greater abundance of Blautia

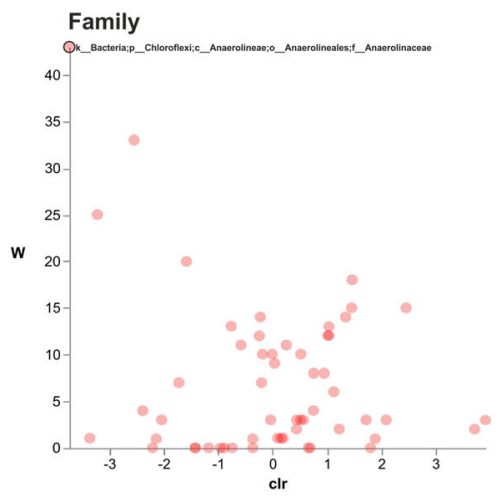

Fig. 5 Volcano plot representation of ANCOM analyses. The horizontal axis is the centered log ratio transformation (clr) representative of the difference in abundance of a given taxonomical unit between Simmental cows and Simmental heifers at the family (red dots), genus (green dots), and species (blue dots) level. The W statistic indicates the value of the statistical test corresponding to the number of times the null
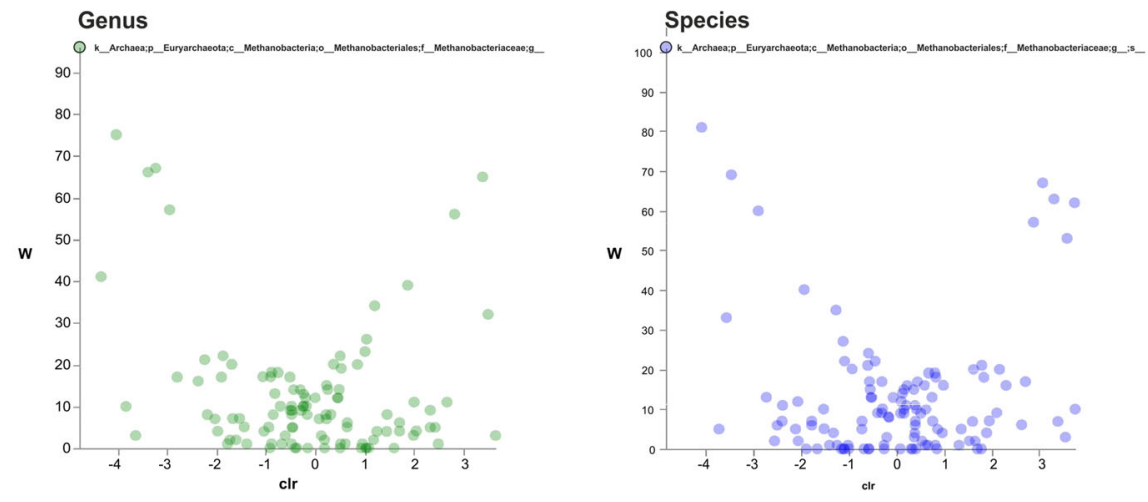

hypothesis was rejected for each taxonomical class. The family, genus, and species with relative abundance significantly different (HolmBonferroni corrected $p$ value $<0.05$ ) are evidenced at the top of volcano plot and their taxonomy are reported. A darker shade of color indicates an overlap of OTUs 
and Roseburia which seem to be correlated with a corn-rich diet and are involved in the production of butyrate; the latter plays a role in the energy source for the mucosa (Kim et al. 2014), similar to Fecalibacterium which promotes the main energy sources for the gut epithelial cells (Pryde et al. 2002). The most representative genera, in all experimental groups, were correlated to digest functions, such as Clostridium, Eubacterium, Paeniclostridium, Romboutsia, and Turicibacter as related to diet composition and probably to physiological traits (Fan et al. 2017; Gerritsen et al. 2017; Xin et al. 2019).

Results of $\alpha$-diversity analyses showed a significant difference between HFH and HFC, which is probably related to animal categories and diet composition. Finally, the OTU table was normalized according to the OTU abundance across samples, and the results were clustered as the heatmap to provide a better pattern across the experimental groups (Fig. 2). There was high difference of the clustered OTUs across the experimental groups; nevertheless, we cannot compare the breeds due to different farming conditions (diet and management conditions).

The PCoA showed the presence of two distinct groups (Fig. 3), which represent heifers and cows; indeed, the microbiome seems to be notably different between these two animal categories. We can indirectly justify the microbiome difference between heifers and cows, since diet (Callaway et al. 2010; Fan et al. 2017; Xin et al. 2019) and lactation (AlZahal et al. 2017) are important factors for the determination of bacteriological composition of fecal community. The variation of data could be explained as there is evidence in scientific literature indicating that the composition of fecal microbiome is completely different from the rumen and the differences between the taxonomical groups were less in the fecal samples compared with rumen (Azad et al. 2019). The differences between HFH and HFC and between SIH and SIC are strongly marked, especially with regard to some microbial populations, such as Tenericutes, Actinobacteria, and Bacteroidetes between HFC and HFH, and Chloroflexi and Euryarchaeota between SIC and SIH. This variability can be explained by diet composition in relation to the physiological status of the animals. However, the statistical analysis identified families, genus, and species present just in a specific category and breed. The variability of feces microbiome between the animals of the same experimental group was low, denoting a good reproducibility within samples. Interestingly the taxonomic class seems to cluster between heifers and cows of the 2 different breeds. Even if we could not perform a direct comparison between breeds, we can note that the trend in cows and heifers is conserved. Therefore, we may hypothesize that this trend is fixed, even when different interactions among breeds and diets are considered. Recently, a specific study reported a genetic link between breed and rumen microbiome community (Sandri et al. 2018); thus, as future perspective, we will investigate the microbiome composition of feces samples from animals of different breeds under the same farming and diet conditions. Finally, microbiome composition is crucial to evaluate feed efficiency, animal welfare, and methane emissions from cattle (Oikonomou et al. 2013; Holmes and Smith 2016; Sandri et al. 2018). Indeed, several alterations of the microbiome can compromise the physiological functions of the animals, altering their production capacity and health. Thus, our study lays the groundwork for future investigations aimed at developing minimally invasive and routine screening system to collect information on microbiome composition at population level. This would open new opportunities to select for more efficient and healthy animals.

Acknowledgments Open access funding provided by Università degli Studi di Padova within the CRUI-CARE Agreement. We thank BMR Genomics service for the NGS analysis. We acknowledge the experimental farm "Lucio Toniolo" (University of Padova, Legnaro, Italy) for providing feces of Simmental cattle, and a commercial farm for providing feces of Holstein-Friesian cattle.

Funding information This work was supported by POR-FESR 20142020 Regione Veneto, NIP project.

\section{Compliance with ethical standards}

Conflict of interest The authors declare that they have no conflict of interest.

Ethical standards The collection of the fecal samples was performed during routine health monitoring of animals by authorized veterinarians of the Breeds Association of Veneto Region (Italy) in compliance with the European rules (Council Regulation (EC) No. 1/2005).

Open Access This article is licensed under a Creative Commons Attribution 4.0 International License, which permits use, sharing, adaptation, distribution and reproduction in any medium or format, as long as you give appropriate credit to the original author(s) and the source, provide a link to the Creative Commons licence, and indicate if changes were made. The images or other third party material in this article are included in the article's Creative Commons licence, unless indicated otherwise in a credit line to the material. If material is not included in the article's Creative Commons licence and your intended use is not permitted by statutory regulation or exceeds the permitted use, you will need to obtain permission directly from the copyright holder. To view a copy of this licence, visit http://creativecommons.org/licenses/by/4.0/.

\section{References}

AlZahal O, Li F, Walker ND, McBride BW (2017) Factors influencing ruminal bacterial community diversity and composition and microbial fibrolytic enzyme abundance in lactating dairy cows with a focus on the role of active dry yeast. J Dairy Sci 100(6):4377-4393

Anderson MJ (2005) PERMANOVA: a FORTRAN computer program for permutational analysis of variance. Department of Statistics, University of Auckland

Azad E, Derakhshani H, Forster RJ, Gruninger RJ, Acharya S, McAllister TA, Khafipour E (2019) Characterization of the rumen and fecal 
microbiome in bloated and non-bloated cattle grazing alfalfa pastures and subjected to bloat prevention strategies. Sci Rep 9(1):1-13

Borsanelli AC, Lappin DF, Viora L, Bennett D, Dutra IS, Brandt BW, Riggio MP (2018) Microbiomes associated with bovine periodontitis and oral health. Vet Microbiol 218:1-6

Callaway TR, Dowd SE, Edrington TS, Anderson RC, Krueger N, Bauer $\mathrm{N}$ et al (2010) Evaluation of bacterial diversity in the rumen and feces of cattle fed different levels of dried distillers grains plus solubles using bacterial tag-encoded FLX amplicon pyrosequencing. J Anim Sci 88(12):3977-3983

Deusch S, Camarinha-Silva A, Conrad J, Beifuss U, Rodehutscord M, Seifert J (2017) A structural and functional elucidation of the rumen microbiome influenced by various diets and microenvironments. Front Microbiol 8:1605

Durso LM, Wells JE, Harhay GP, Rice WC, Kuehn L, Bono JL et al (2012) Comparison of bacterial communities in faeces of beef cattle fed diets containing corn and wet distillers' grain with solubles. Lett Appl Microbiol 55(2):109-114

Fan P, Liu P, Song P, Chen X, Ma X (2017) Moderate dietary protein restriction alters the composition of gut microbiota and improves ileal barrier function in adult pig model. Sci Rep 7:43412

Gerritsen J, Hornung B, Renckens B, van Hijum SA, dos Santos VAM, Rijkers GT et al (2017) Genomic and functional analysis of Romboutsia ilealis CRIBT reveals adaptation to the small intestine. PeerJ 5:e3698

Holmes DE, Smith JA (2016) Biologically produced methane as a renewable energy source. In Advances in applied microbiology (Vol. 97, pp. 1-61). Academic Press

Hook SE, Wright ADG, McBride BW (2010) Methanogens: methane producers of the rumen and mitigation strategies. Archaea

Kim M, Wells JE (2016) A meta-analysis of bacterial diversity in the feces of cattle. Curr Microbiol 72(2):145-151

Kim M, Kim J, Kuehn LA, Bono JL, Berry ED, Kalchayanand N et al (2014) Investigation of bacterial diversity in the feces of cattle fed different diets. J Anim Sci 92(2):683-694

Kim M, Park T, Yu Z (2017) Metagenomic investigation of gastrointestinal microbiome in cattle. Asian Australas J Anim Sci 30(11):1515

Klevenhusen F, Petri RM, Kleefisch MT, Khiaosa-ard R, Metzler-Zebeli BU, Zebeli Q (2017) Changes in fibre-adherent and fluid-associated microbial communities and fermentation profiles in the rumen of cattle fed diets differing in hay quality and concentrate amount. FEMS Microbiol Ecol 93(9)

Kuczynski J, Stombaugh J, Walters WA, González A, Caporaso JG, Knight R (2012) Using QIIME to analyze 16S rRNA gene sequences from microbial communities. Curr Protoc Microbiol 27(1):1E-5E

Mende DR, Waller AS, Sunagawa S, Järvelin AI, Chan MM, Arumugam $\mathrm{M}$ et al (2012) Assessment of metagenomic assembly using simulated next generation sequencing data. PLoS One 7(2)
Nyonyo T, Shinkai T, Mitsumori M (2014) Improved culturability of cellulolytic rumen bacteria and phylogenetic diversity of culturable cellulolytic and xylanolytic bacteria newly isolated from the bovine rumen. FEMS Microbiol Ecol 88(3):528-537

Oikonomou G, Teixeira AGV, Foditsch C, Bicalho ML, Machado VS, Bicalho RC (2013) Fecal microbial diversity in pre-weaned dairy calves as described by pyrosequencing of metagenomic 16S rDNA. Associations of Fecalibacterium species with health and growth. PloS one, 8(4).

Oksanen J, Blanchet FG, Kindt R, Legendre P, Minchin PR, O'hara RB, et al. (2017) Vegan: Community Ecology Package. R package version $2.3-0 ; 2015$.

Pryde SE, Duncan SH, Hold GL, Stewart CS, Flint HJ (2002) The microbiology of butyrate formation in the human colon. FEMS Microbiol Lett 217(2):133-139

Rice WC, Galyean ML, Cox SB, Dowd SE, Cole NA (2012) Influence of wet distillers grains diets on beef cattle fecal bacterial community structure. BMC Microbiol 12(1):25

Sandri M, Licastro D, Dal Monego S, Sgorlon S, Stefanon B (2018) Investigation of rumen metagenome in Italian Simmental and Italian Holstein cows using a whole-genome shotgun sequencing technique. Ital J Anim Sci 17(4):890-898

Shanks OC, Kelty CA, Archibeque S, Jenkins M, Newton RJ, McLellan SL et al (2011) Community structures of fecal bacteria in cattle from different animal feeding operations. Appl Environ Microbiol 77(9): 2992-3001

Shepherd ML, Swecker WS Jr, Jensen RV, Ponder MA (2012) Characterization of the fecal bacteria communities of forage-fed horses by pyrosequencing of $16 \mathrm{~S}$ rRNA V4 gene amplicons. FEMS Microbiol Lett 326(1):62-68

Sulak M, Sikorova L, Jankuvova P, Javorsky P, Pristas P (2012) Variability of Actinobacteria, a minor component of rumen microflora. Folia Microbiol. https://doi.org/10.1007/s12223-012-0140-7

Takahashi S, Tomita J, Nishioka K, Hisada T, Nishijima M (2014) Development of a prokaryotic universal primer for simultaneous analysis of Bacteria and Archaea using next-generation sequencing. PLoS One 9(8)

Team, R. C. (2015). R: A language and environment for statistical computing.

Tringe SG, Hugenholtz P (2008) A renaissance for the pioneering 16S rRNA gene. Curr Opin Microbiol 11(5):442-446

Xin JW, Chai Z, Zhang C, Cao H, Zhu Y, Zhang Q et al (2019) Comparing the microbial community in four stomach of dairy cattle, yellow cattle and three yak herds in Qinghai-Tibet Plateau. Front Microbiol 10:1547

Publisher's note Springer Nature remains neutral with regard to jurisdictional claims in published maps and institutional affiliations. 